\title{
VOGT-KOYANAGI-HARADA DISEASE IN A CHILD: A RARE CASE
}

\author{
Isabela Oliveira Vieira da Silva ${ }^{1, *}$, Nabila Triântala Bellezzia de Salles', Paula Consoline Micheletto', Antonio Lucas Lima \\ Rodrigues' ${ }^{1}$, Maraísa Centeville', Roberto Marini', Simone Appenzeller ${ }^{1}$
}

1. Universidade Estadual de Campinas, Campinas (SP), Brazil.

*Corresponding author: isa_ovs@hotmail.com

\section{BACKGROUND}

Vogt-Koyanagi-Harada disease (VKHD) is a rare multisystemic and inflammatory autoimmune disease. It is mediated by $T$ lymphocytes against melanocyte proteins in susceptible individuals. It mainly affects black or mixed women in the fourth decade of life. It has four phases: prodromal, uveitic, convalescent and recurrent. It is characterized by bilateral uveitis and hypoacusis, meningismus, poliosis and vitiligo. The diagnosis is clinical, based on findings on retinography, ocular ultrasound and fluorescein angiography. Initial treatment is based on corticosteroid therapy and switched to immunomodulators in refractory cases. The main complications are cataracts, glaucoma, retinal fibrosis and subretinal neovascular membrane.

\section{CASE REPORT}

Patient, 12 years old, male, white, sought an ophthalmology emergency room with complaint of conjunctival hyperemia, decreased visual acuity on the right and headache for a month. The retinoscopy showed papilledema and bilateral neuritis. A cerebrospinal fluid (CSF) analysis with manometry, head computerized tomography (CT) and electroencephalogram (EEG) were made. Cerebrospinal fluid showed pleocytosis, manometry was normal and EEG and CT showed no signs of intracranial hypertension. Magnetic resonance imaging (MRI) was performed and showed chorioretinitis and uveoscleral inflammatory signs. With the hypothesis of posterior scleritis triggered by Herpes virus infection and Harada-like syndrome, the patient was prescribed empirical acyclovir for 10 days followed by prednisolone with slow tapering dose and evolved with improvement of symptoms in a year. After remaining asymptomatic for 17 months, the patient represented the initial symptoms, the hypothesis of VKHD was made and methylprednisolone pulse therapy was prescribed. The patient was then referred to our service to evaluate immunosuppression. Subcutaneous methotrexate was initiated, later transposed to oral. During 18 months of use of methotrexate the patient presented a great evolution, currently showing no signs of disease activity, so we opted for suspension of medication.

\section{CONCLUSION}

This case shows the difficulty of diagnosing a rare disease, specially since our patient differs from epidemiological standards in addition to the difficulty on performing genetic tests. Although our patient is male and young, the natural course of the disease could be observed with the uveitic, convalescent and recurrent phases. The prodromal phase may have gone unnoticed since it shows nonspecific symptoms. Our patient did not present otorhinolaryngological and dermatological symptoms, nor complications. The diagnosis of VKHD is clinical and this study aims to highlight the importance of early diagnosis to properly treat and avoid complications. 